\title{
Chronic Ulcers in Thromboangiitis Obliterans (Buerger's Disease): Updating Epidemiology, Physiopathology, and Bosentan-A Novel Strategy of Therapy
}

\author{
Ignacio López de Maturana, Javier Rodriguez, Carmen González, Silvia Bleda, \\ Joaquin de Haro, and Francisco Acin \\ Vascular Surgery and Angiology Department, Hospital Universitario Getafe, Ctra Toledo Km 12,500, 28905 Madrid, Spain \\ Correspondence should be addressed to Silvia Bleda; silbleik@yahoo.es
}

Received 31 August 2012; Accepted 28 January 2013

Academic Editor: Marisa Herson

Copyright (C) 2013 Ignacio López de Maturana et al. This is an open access article distributed under the Creative Commons Attribution License, which permits unrestricted use, distribution, and reproduction in any medium, provided the original work is properly cited.

\begin{abstract}
Thromboangiitis obliterans (TAO) or Buerger's disease is associated with both distal ulcers in the extremities and the possibility of amputation. The only treatment that has been shown to be effective in TAO is complete abstention from smoking. In spite of this, the disease progresses in up to 30 percent of cases and finally results in limb amputation. Only a few pharmacological and surgical options are available to date to improve healing ulcers in TAO. The efficacy of prostaglandin analogues is controversial. This paper summarizes the current evidence for medical treatment with bosentan in chronic ulcers in TAO patients. These available data up to date allow us to conclude that the beneficial effects of bosentan on improving endothelial function, inflammatory processes, and selective vasodilatation of damaged vessels result in a clinical enhancement regarding healing and preventive digital ulcers in such patients. In any case, these promising findings have to be confirmed with larger randomised trials.
\end{abstract}

\section{Introduction}

Thromboangiitis obliterans (TAO) or Buerger's disease is a thrombotic, occlusive, and nonatherosclerotic segmental vasculitis that affects small- and medium-sized arteries and veins which may involve distal vessel of upper and lower extremities. As a vasculitis, it is characterized by inflammation and fibrinoid necrosis of blood vessel walls.

Classically, various mechanisms have been implicated in its etiopathogenesis including cell-mediated inflammation, immune complex-mediated inflammation, and autoantibody-mediated inflammation [1]. Recently, novel pathways have been described in physiopathology of the disease, though not completely well known. The endothelin-1 (ET-1) has been associated in these etiological processes, which can induce to endothelial cell activation causing complications such as vessel occlusion and tissue destruction [2].

ET-1 is a potent vasoconstrictor peptide, which exerts its action by targeting two transmembrane receptors (ETA and ETB). ET-1 facilitates the proliferation of vascular smooth muscle cells, promotes monocytes via activation of the ETA, and contributes to matrix remodelling leading to the abnormal thickening of vessel walls [1-3]. Raised levels of ET-1 have been described in different kind of systemic vasculitis as mixed cryoglobulinemia, secondary Raynaud's phenomenon [2], acute phase of Henoch-Schölein purpura, early stages of giant cell arteritis [1], Takayasu's arteritis, and Buerger's disease [3]. This finding supports that ET-1 may act as marker of vascular damage $[2,3]$. Other nonvasculitic entities as rheumatoid arthritis, systemic lupus erythematous, systemic sclerosis, pulmonary hypertension, or artherosclerosis have been also associated with high levels of ET-1 and have been related to vascular injury. Although data are limited, there is evidence suggesting that ET-1 plays a role in the clinical manifestations of vasculitis. Accordingly, blockade of ET-1 could therefore be of therapeutic benefit in these diseases.

TAO usually occurs in people around the age of 45 and is more frequent in male smokers. In the general population of USA, its incidence has declined as tobacco use has also declined. However, as a consequence of 
the increase in smoking between women, an increase in the incidence of TAO has been observed in the last 20 years [46]. Intermittent claudication and, in more advanced cases, pain at rest are the predominant clinical symptoms. Distal ischaemic lesions (trophic and ulcerations) are frequently observed by means of physical examination. Clinical course is characterised by alternating periods of exacerbation with periods of remission. Angiographic studies reveal a distal and segmental involvement of the vasculature of the extremities. Recanalisation is frequently demonstrated, showing a typical image (corkscrew collateral vessels) [7]. Skin disorders such as migrating phlebitis or Raynaud-like colour changes may be associated with TAO.

Tobacco use is strongly connected to the onset, progression, and prognosis of this disease. In fact, just less than $5 \%$ of patients are nonsmokers. In those small percentages of nonsmoker patients, the mechanisms that trigger the disease are not completely known. Published hypothesis state that it might be induced by cold, frostbite, traumatism of extremities, or even abuse of sympathomimetic drugs [8].

Interestingly, an impaired endothelium-dependent vasodilatation in the peripheral vasculature, even in the nondiseased limbs, has been shown in patients with TAO [9]. Although, mild perturbations in clotting have been described, there is no evidence suggesting that hypercoagulability or fibrinolytic abnormalities play a major role in the ethiopatogenia of this disease [8]. Moreover, various investigations have been also carried out with the aim of indentifying an autoimmune mechanism responsible for TAO. However, the abnormalities found have proved to be nonspecific and have not been completely confirmed [8].

Therefore, giving up smoking is the most important therapeutic measure in TAO patients [10]. In fact, it leads to dramatic improvement of the symptoms and lesions. Otherwise, drugs used to manage TAO, prostacyclin (PGI2) or its analogues (iloprost, beraprost, trepostinil sodium), aspirin, or streptokinase (as a thrombolytic) have shown an uncertain efficacy. On the other hand, revascularization by means of a bypass surgery or endovascular procedure is usually not possible as a consequence of the predominantly diffuse and distal location of the lesions in the veins and arteries involved. The fact that TAO is highly associated with both distal ulcers in the extremities and the possibility of amputation leads frequently to involve in social problems and a worsening in the quality of life of the affected patients [11]. Added to the fact that only a few pharmacological and surgical options (of controversial efficacy) are available to date, new therapeutic options with a higher efficacy than the current ones are clearly needed in order to properly manage patients affected by TAO.

ET-1 receptor blockade may be used as a therapeutic target for improvement in TAO patients. Pharmacologic ET1 receptor blockade may be single (ETA or ETB) or dual (both, ETA and ETB) [12]. Bosentan is a dual ET-1 receptor antagonist, administered orally, which is approved by the European Union to treat pulmonary arterial hypertension in systemic sclerosis patients and to prevent the occurrence of new digital ulcers in systemic sclerosis patients with ongoing digital ulcers. Recent investigations have suggested that bosentan could have a role in healing ongoing digital ulcers in TAO [13-18].

This paper summarizes the current evidence for medical treatment with bosentan in chronic ulcers in vasculitis, especially in TAO patients.

\section{Effect of Bosentan on Microcirculation Physiopathology}

ET-1 is an endothelium-derived peptide, which is involved in the regulation of vascular function under normal physiologic conditions [19]. It plays a key role in vascular pathologies by exerting various deleterious effects. These include hypertrophy of vascular smooth muscle cells, cellular proliferation, fibrosis, increase of vascular permeability, activation of leukocytes, and induction of cytokine and adhesion molecule expression $[19,20]$. Moreover, ET-1 is the most potent natural vasoconstrictive mediator. It has been demonstrated that its exogenous administration in healthy volunteers produces a marked dose-dependent reduction of the blood flow [21].

The effects of ET-1 are transmitted upon binding 2 cognate receptors, ETA and ETB, which are mainly expressed on endothelial cells (ET-B), smooth muscle cells, and fibroblasts $[19,20]$. Elevated circulating levels of ET-1 have been repeatedly observed in scleroderma, as well as in various other pathologies in which the vascular endothelium is involved [22]. It has been also detected an increase in plasma levels of ET-1 in situations of acute or chronic limb ischemia, chronic and acute coronary syndromes, acute renal failure, and stroke $[23,24]$. Nevertheless, the role of ET-1 activity as a causal factor of endothelial dysfunction and/or damage or an epiphenomenon remains not completely clear [22, 25]. Experimental studies in animal models of hypertension [26, 27] and atherosclerosis [28] have shown an improvement in the endothelial function of large arteries following shortterm administration of endothelin receptor antagonists. Any case, these data point that some of the endothelin-mediated deleterious effects on the vasculature may be reversible.

Bosentan, an oral dual ET-1 receptor antagonist, can exert a selective vasodilator effect on the vascular bed. Its efficacy has been demonstrated, with a favourable safety profile, in two randomised controlled clinical trials, RAPIDS-1 and RAPIDS-2, for the treatment and prevention of digital ulcers in patients with systemic sclerosis $[13,29]$. The results of such trials suggest that it may be beneficial for the treatment of Raynaud phenomenon. There is evidence that bosentan exert a selective vasodilator and anti-inflammatory effects in patients affected by TAO, comparable to the effects observed in connective tissue diseases.

Several studies have shown that can improve endothelial function after 4 weeks of treatment, indirectly demonstrated by the increasing of the flow-mediated dilation (FMD) measurements in the brachial artery in patients with systemic sclerosis, diabetes mellitus, microalbuminuria, and peripheral artery disease $[17,24,30]$.

Meanwhile, Nitric oxide (NO) is considered to be another reliable marker and is involved in the homeostasis of endothelial function [31]. Endothelial dysfunction appears 
as an early change in the onset stages of vasculitis [32]. An increase in ET-1 activity has also been associated to an inhibition of NO synthesis [30]. Recent investigations have suggested that an improvement in endothelial function would be achieved by enhanced NO production. Thus, treatment with bosentan could improve NO synthesis in patients with vasculitis by inhibition of the ET-1 [30]. These data allow us to hypothesize that the improvement of endothelial dysfunction, after bosentan treatment, may not only be associated with hemodynamic changes, proinflammatory processes, or activated endothelium effects, but rather may be due to the enhancement of NO production following inhibition of ET1 , as has previously been seen in pulmonary hypertension $[33,34]$. These findings prove that the endothelin receptor system is an important molecular pathway that is directly involved in certain reversible aspects of vascular injury.

\section{Efficacy of Bosentan on Chronic Ulcers Treatment in Buerger's Disease}

Up to date, the only treatment that has been shown to be effective in TAO is complete abstention from smoking. Both clinical improvement and complete healing of the ulcers have been achieved in the majority of patients after giving up smoking. In spite of this, the disease progresses in up to 30 percent of cases and finally results in multiple limb amputation [35]. Furthermore, giving up smoking is achieved in a very low number of these patients, inferior to $30 \%$ in some studies [17]. This unsatisfactory rate, in accordance with previous reports, highlights the fact that it is extremely difficult for patients, who are heavy smokers, to give up smoking despite having strongly been advised to do so, as well as received full information about the benefits of giving up smoking, especially in terms of avoiding amputations [36].

Only a few pharmacological and surgical options (of controversial efficacy) are available to date to improve healing ulcers in TAO [8]. Vasodilators, antiplatelet agents, anticoagulants, and corticosteroids appear to be of no use [37]. Prostaglandin analogues are beneficial when administered intravenously [38], although their efficacy is controversial on oral administration [39]. A randomised clinical trial of intravenous iloprost versus aspirin [38] has shown that healing of ulcers is higher in patients who have received treatment with intravenous prostaglandins. Nonetheless, in other randomised trials, an oral formulation of iloprost has not been better than placebo with regard to this outcome [39]. Therefore, the efficacy results shown by prostacyclin analogues when used for the management of TAO are far from satisfactory.

Meanwhile, sympathectomy may alleviate the pain and improves superficial ulcers, but it does not prevent or reduce the number of amputations [37]. Surgical revascularization is not usually feasible because of the diffuse and segmental character of the disease [37]. Thus, new therapeutic options with a higher efficacy than the current ones are clearly needed in order to properly manage patients affected by TAO. In any case, the characteristics of this disease, the low incidence, and the lack of effective treatments that improve the course of the disease or correct the cause contributed to serious ethical difficulties in carrying out large prospective studies that confirm the benefits and further definitive assessment in comparative randomised trials of the efficacy of novel therapy in this particular disease.

There are few articles published regarding the treatment of TAO with bosentan. However, they have shown that bosentan therapy is associated with several clinical and endothelial function-related outcomes in patients with TAO, which may be promising.

The anti-inflammatory, antifibrotic, and selective vasodilator properties of bosentan have been shown to alleviate pain at rest and reduce the size of ischaemic ulcers caused by damage mainly to the microcirculation. Recently, a single centre clinical study has been published, where 12 patients (13 extremities) previously diagnosed with TAO received treatment with bosentan in a compassionate use programme [17]. Bosentan therapy consisted of a month treatment with $62.5 \mathrm{mg}$ twice a day followed by a double dose after the first month. The full-dose regimen was maintained for the following three months or until total healing of the ulcers. Prior to the treatment with bosentan, 10 of 12 patients hve previously been treated with a 21 days prostaglandin regimen, 3 had been undergone revascularizing procedures, and 3 patients had a lumbar sympathectomy. Clinical improvement was observed in 12 extremities (92\%) treated, while only 1 extremity required major amputation below the knee. 10 extremities (77\%) achieved complete clinical therapeutic success (healing or complete pain relief). A minor amputation of one toe was performed with conservation of the extremity. Also, a statistical improve of the endothelial function that was assessed by means of the FMD was observed.

Several case reports have been also published in the literature. All of them provide information on TAO patients with a history of insidious necrotic ulcers with poor outcomes despite smoking cessation and conventional medical treatment, including intravenous prostaglandins [18, 40, 41]. Their results show that treatment with bosentan is able to obtain a favourable clinical response with healing of ulcers, as well as the disappearing of the rest pain. Furthermore, most patients remained asymptomatic for six months after treatment cessation. Therefore, beneficial effects of bosentan in TAO patients are not only during the acute phase of ulcers and rest pain, but also they extend over time.

Although these results are from a small study and case reports and are not comparable with those from randomised trials, they seem to be hopeful.

A possible explanation for the bosentan pharmacodynamic effect has been related on its capacity of improving endothelial function based on the endothelial function impairment observed in patients with peripheral arterial disease in general [42] and in TAO patients in particular after treatment [9]. Moreover, an elevated serum ET-1 level has been observed in patients with TAO, supporting a possible mechanistic explanation of the clinical benefit of bosentan in these patients $[3,43]$. Additionally, bosentan can exert a selective vasodilatory and anti-inflammatory effect on the vascular bed in patients affected by TAO, comparable to 
the effects observed in connective tissue diseases such as scleroderma with the added complication of digital ulcers.

Summarizing, bosentan should be further investigated with regard to TAO patient management. The hypothesis that bosentan treatment in TAO patients results in an improvement of clinical, angiographic, and endothelial function outcomes is supported by the results of a small pilot study and several case reports that have been recently published. However, larger prospective studies and comparative randomised trials are needed to confirm them.

\section{Treatment of Other Types of Vasculitis Digital Ulcers with Bosentan}

The use of bosentan in Europe is approved for the treatment of pulmonary arterial hypertension and for digital ulcers (DUs) due to systemic sclerosis (SSc). The key sources of evidence for the use of bosentan in the management of digital ulcers in scleroderma are RAPIDS- 1 and RAPIDS- 2 trials $[28,29]$.

RAPIDS-1 is a randomized, prospective, placebocontrolled, and double-blind study of 122 patients with confirmed diagnosis of SSc. The primary outcome measure in this trial was the number of new DUs developed during the 16 week study period. Secondary assessment included healing of existing DUs. This trial demonstrated a significant beneficial difference between patients with bosentan compared to patients with placebo in the primary endpoint regarding to the appearance of new DUs. This difference was greater in patients who had ulcers at baseline $(63 \%)$ and in those with diffuse disease. Nevertheless, no differences were found between placebo and bosentan in the time of complete or partial healing of DUs [44].

RAPIDS-2 is a randomized, double-blind, placebocontrolled trial with 188 SSc patients with at least one active DU [29]. In this trial the two primary endpoints were the number of new DUs and the time of healing of the DUs. Over 24 weeks, bosentan treatment was significantly associated with a $30 \%$ reduction in the number of new DUs compared with placebo. This effect was greater in patients who entered the trial with more DUs. Once again, there was no difference between treatments in the healing rate of DUs [29].

In both trials, bosentan treatment has demonstrated its ability to reduce the occurrence of new DUs in patient with SSc. However, this treatment has no effect on DU period of healing. Besides, there are published small series of patients that also show the beneficial effects of bosentan on preventing DUs, confirming the evidence obtained from RAPIDS studies $[14,16,45-47]$. An open-label study of 15 patients with SSc and DUs using bosentan for a median period of 24 months has revealed that bosentan is safe and effective in these patients [45]. Tsifetaki et al. have reported the longest prospective study (until 4 years) evaluating the number of healed DUs and new ulcer formation in 30 patients with SSc. Their results have showed that healed DUs occurred in $65 \%$ of treated patients [16].

There are also references in the literature regarding successful treatment with bosentan of refractory ulcers secondary to other pathologies. Bosentan treatment has been effective in healing of refractory DUs in patients with systemic lupus erythematous $[48,49]$, as well as in DUs due to Werner syndrome [50].

In addition, bosentan has been also used in paediatric patients. Studies in children with pulmonary hypertension have demonstrated its safety [51]. In this group of patients bosentan has also been effective to treat digital necrosis secondary to polyarteritis nodosa [52] and SSc [53].

In conclusion, the known beneficial effect of bosentan in the prevention of DUs secondary to SSc must be added to the promising healing effect on ulcers due to other pathologies, like systemic lupus erythematosus, polyarteritis nodosa, or Werner syndrome. Bosentan may be taken into account as a treatment option in these vasculitis diseases. Nonetheless, it is necessary to design prospective, randomized, and controlled trials to confirm these amazing results drawn from open-label or noncontrolled studies.

\section{Summary and Conclusions}

Endothelin-1 has been associated to vascular damage responsible for vasculitis and plays a key role in its clinical manifestations.

Focused on TAO, a vasculitis that affects small- and medium-sized arteries and veins of both upper and lower extremities, distal ischaemic lesions, and digital necrotic ulcers are frequently observed. Major amputation rate among these patients is not negligible. Furthermore, increased levels of ET-1 have been proved in TAO patients. Thus, ET-1 receptor antagonists, as bosentan, should be considered as a useful treatment option in this disease.

Initial results from open-label, nonparallel groups controlled studies or case reports published articles show promising efficacy of bosentan for treatment and prevention of digital ulcers in TAO with a favourable safety profile.

This efficacy can be justified by a selective vasodilator effect on the vascular bed, improving vascular permeability in digital ischemic ulcers, and restitution of endothelial function by increasing NO levels.

The anti-inflammatory, antifibrotic, and selective vasodilator properties of bosentan have been demonstrated to alleviate pain at rest and reduce the size of ischemic ulcers due to TAO. Beneficial effects of bosentan in TAO patients have been also reported to extend over time.

On the other hand, Bosentan has been found to decrease the number of new digital ulcers, although no significant effect in the healing period duration has been observed in patients with SSc. Moreover, Bosentan have been also stated to be effective in the healing of refractory ulcers due to systemic lupus erythematous, Werner syndrome, or polyarteritis nodosa.

Lastly, in any case, Bosentan should be further investigated in TAO and vasculitis patient management. To confirm these promising findings, larger controlled randomised trials with a control group are needed. In the meantime, bosentan should be considered as a hopeful investigational agent for treating these patients. 


\section{References}

[1] L. -Guillevin and T. Dörner, "Vasculitis: mechanisms involved and clinical manifestations," Arthritis Research and Therapy, vol. 9, supplement 2, p. S9, 2007.

[2] C. Ferri, A. Latorraca, G. Catapano et al., "Increased plasma endothelin-1 immunoreactive levels in vasculitis: a clue to the use of endothelin-1 as a marker of vascular damage?" Journal of Hypertension, vol. 11, no. 5, pp. S142-S143, 1993.

[3] K. Kanno, Y. Hirata, F. Numano et al., "Endothelin-1 and vasculitis," The Journal of the American Medical Association, vol. 264, no. 22, article 2868, 1990.

[4] A. Szuba and J. P. Cooke, "Tromboangiitis obliternas an uptodate on Berguer's disease," The Western Journal of Medicine, vol. 168, pp. 255-260, 1998.

[5] J. W. Olin, J. R. Young, R. A. Graor, W. F. Ruschhaupt, and J. R. Bartholomew, "The changing clinical spectrum of thromboangiitis obliterans (Buerger's disease)," Circulation, vol. 82, no. 5, supplement, pp. IV3-IV8, 1990.

[6] Y. Yorukoglu, E. Ilgit, M. Zengin, K. Nazliel, E. Salman, and E. Yucel, "Thromboangiitis obliterans (Buerger's disease) in women (a reevaluation)," Angiology, vol. 44, no. 7, pp. 527-532, 1993.

[7] T. Yoshimuta, K. Akutsu, T. Okajima, Y. Tamori, Y. Kubota, and S. Takeshita, "Corkscrew collaterals in Buerger's disease," Canadian Journal of Cardiology, vol. 25, no. 6, article 365, 2009.

[8] X. Puéchal and J. N. Fiessinger, "Thromboangiitis obliterans or Buerger's disease: challenges for the rheumatologist," Rheumatology, vol. 46, no. 2, pp. 192-199, 2007.

[9] S. Makita, M. Nakamura, H. Murakami, K. Komoda, K. Kawazoe, and K. Hiramori, "Impaired endothelium-dependent vasorelaxation in peripheral vasculature of patients with thromboangiitis obliterans (Buerger's disease)," Circulation, vol. 94, no. 9, supplement, pp. II211-II215, 1996.

[10] L. T. Cooper, S. S. Henderson, K. V. Ballman et al., "A prospective, case-control study of tobacco dependence in thromboangiitis obliterans (Buerger's disease)," Angiology, vol. 57, no. 1, pp. 73-78, 2006.

[11] T. Ohta, H. Ishioashi, M. Hosaka, and I. Sugimoto, "Clinical and social consequences of Buerger disease," Journal of Vascular Surgery, vol. 39, no. 1, pp. 176-180, 2004.

[12] S. W. Watts, "Endothelin receptors: what's new and what do we need to know?" The American Journal of Physiology, vol. 298, no. 2, pp. R254-R260, 2010.

[13] D. Launay, E. Diot, E. Pasquier et al., "Bosentan for treatment of active digital ulcers in patients with systemic sclerosis," Presse Medicale, vol. 35, no. 4, pp. 587-592, 2006.

[14] M. Funauchi, K. Kishimoto, H. Shimazu et al., "Effects of bosentan on the skin lesions: an observational study from a single center in Japan," Rheumatology International, vol. 29, no. 7, pp. 769-775, 2009.

[15] M. T. Riccardi, A. Chialà, F. Iannone, V. Grattagliano, M. Covelli, and G. Lapadula, "Treatment of digital ulcers in systemtic sclerosis with endothelin-1 receptor antagonist (bosentan)," Reumatismo, vol. 59, no. 2, pp. 135-139, 2007.

[16] N. Tsifetaki, V. Botzoris, Y. Alamanos, E. Argyriou, A. Zioga, and A. A. Drosos, "Bosentan for digital ulcers in patients with systemic sclerosis: a prospective 3-year followup study," Journal of Rheumatology, vol. 36, no. 7, pp. 1550-1551, 2009.

[17] J. de Haro, F. Acin, S. Bleda, C. Varela, and L. Esparza, "Treatment of thromboangiitis obliterans (Buerger's disease) with bosentan," BMC Cardiovascular Disorders, vol. 12, article 5, 2012.

[18] J. de Haro, A. Florez, J. L. Fernandez, and F. Acin, "Treatment of Buerger disease (thromboangiitis obliterans) with bosentan: a case report," BMJ Case Reports, vol. 2009, 2009.

[19] M. D. Mayes, "Endothelin and endothelin receptor antagonists in systemic rheumatic disease," Arthritis and Rheumatism, vol. 48, no. 5, pp. 1190-1199, 2003.

[20] N. H. S. Kim and L. J. Rubin, "Endothelin in health and disease: endothelin receptor antagonists in the management of pulmonary artery hypertension," Journal of Cardiovascular Pharmacology and Therapeutics, vol. 7, no. 1, pp. 9-19, 2002.

[21] J. G. Clarke, N. Benjamin, S. W. Larkin, D. J. Webb, G. J. Davies, and A. Maseri, "Endothelin is a potent long-lasting vasoconstrictor in men," The American Journal of Physiology, vol. 257, no. 6, pp. H2033-H2035, 1989.

[22] E. L. Schiffrin, "Role of endothelin-1 in hypertension and vascular disease," The American Journal of Hypertension, vol. 14, pp. 83S-89S, 2001.

[23] J. C. S. Tsui and M. R. Dashwood, "A role for endothelin-1 in peripheral vascular disease," Current Vascular Pharmacology, vol. 3, no. 4, pp. 325-332, 2005.

[24] J. de Haro Miralles, A. F. Gónzalez, C. Varela Casariego, and F. A. García, "Onset of peripheral arterial disease: role of endothelin in endothelial dysfunction," Interactive CardioVascular and Thoracic Surgery, vol. 10, no. 5, pp. 760-765, 2010.

[25] D. B. Cines, E. S. Pollak, C. A. Buck et al., "Endothelial cells in physiology and in the pathophysiology of vascular disorders," Blood, vol. 91, no. 10, pp. 3527-3561, 1998.

[26] M. Barton, L. V. D’Uscio, S. Shaw, P. Meyer, P. Moreau, and T. F. Lüscher, "ET(A) receptor blockade prevents increased tissue endothelin-1, vascular hypertrophy, and endothelial dysfunction in salt-sensitive hypertension," Hypertension, vol. 31, no. 1, pp. 499-504, 1998.

[27] J. P. Spiers, E. J. Kelso, W. F. Siah et al., "Alterations in vascular matrix metalloproteinase due to ageing and chronic hypertension: effects of endothelin receptor blockade," Journal of Hypertension, vol. 23, no. 9, pp. 1717-1724, 2005.

[28] M. Barton, C. C. Haudenschild, L. V. D’Uscio, S. Shaw, K. Munter, and T. F. Luscher, "Endothelin ETA receptor blockade restores $\mathrm{NO}$-mediated endothelial function and inhibits atherosclerosis in apolipoprotein E-deficient mice," Proceedings of the National Academy of Sciences of the United States of America, vol. 95, no. 24, pp. 14367-14372, 1998.

[29] M. Matucci-Cerinic, C. P. Denton, D. E. Furst, M. D. Mayes, V. M. Hsu, P. Carpentier et al., "Bosentan treatment of digital ulcers related to systemic sclerosis: results from the RAPIDS-2 randomised, double-blind, placebo-controlled trial," Annals of the Rheumatic Diseases, vol. 70, no. 1, pp. 32-38, 2011.

[30] P. P. Sfikakis, C. Papamichael, K. S. Stamatelopoulos et al., "Improvement of vascular endothelial function using the oral endothelin receptor antagonist bosentan in patients with systemic sclerosis," Arthritis and Rheumatism, vol. 56, no. 6, pp. 1985-1993, 2007.

[31] R. Joannides, W. E. Haefeli, L. Linder et al., "Nitric oxide is responsible for flow-dependent dilatation of human peripheral conduit arteries in vivo," Circulation, vol. 91, no. 5, pp. 1314-1319, 1995.

[32] Y. Allanore, D. Borderie, P. Hilliquin et al., "Low levels of nitric oxide (NO) in systemic sclerosis: inducible NO synthase 
production is decreased in cultured peripheral blood monocyte/macrophage cells," Rheumatology, vol. 40, no. 10, pp. 10891096, 2001.

[33] M. B. Kahaleh, "Vascular involvement in systemic sclerosis (SSc)," Clinical and Experimental Rheumatology, vol. 22, no. 3, supplement 33, pp. S19-S23, 2004.

[34] R. E. Girgis, H. C. Champion, G. B. Diette, R. A. Johns, S. Permutt, and J. T. Sylvester, "Decreased exhaled nitric oxide in pulmonary arterial hypertension: response to Bosentan therapy," American Journal of Respiratory and Critical Care Medicine, vol. 172, no. 3, pp. 352-357, 2005.

[35] J. W. Olin, “Thomboangiitis obliterans (Buerguer's disease)," The New England Journal of Medicine, vol. 343, no. 12, pp. 864-869, 2000.

[36] A. Szuba and J. P. Cooke, "Thromboangiitis obliterans-an update on Buerger's disease," The Western Journal of Medicine, vol. 168 , no. 4, pp. 255-260, 1998.

[37] M. R. Jaff, “Thromboangiitis obliterans (Buerguer's disease)," Current Treatment Options in Cardiovascular Medicine, vol. 2, no. 3, pp. 205-212, 2000.

[38] J. N. Fiessinger and M. Schäfer, "Trial of iloprost versus aspirin treatment for critical limb ischaemia of thromboangiitis obliterans," The Lancet, vol. 335, no. 8689, pp. 555-557, 1990.

[39] The European TAO Study Group, "Oral iloprost in the treatment of thromboangiitis obliterans (Buerguer's disease): a doubleblind, randomidez, placebo-controlled trial," European Journal of Vascular and Endovascular Surgery, vol. 15, no. 4, pp. 300-307, 1998.

[40] A. Palomo-Arellano, I. Cervigón-González, and L. M. TorresIglesias, "Effectiveness of bosentan in the treatment of ischemic lesions in a case of thromboangiitis obliterans (Buerguer's disease): a case report," Dermatology Online Journal, vol. 17, no. 7, article 4, 2011.

[41] J. A. T. Parra, M. M. Hernández, and M. A. A. López, "Efficacy of bosentan in digital ischemic ulcers," Annals of Vascular Surgery, vol. 24 , no. 5, article 690, 2010.

[42] N. B. Zimmerman, "Occlusive vascular disorders of the upper extremity," Hand Clinics, vol. 9, no. 1, pp. 139-150, 1993.

[43] M. Czarnacki, M. Gacka, and R. Adamiec, "A role of endothelin 1 in the pathogenesis of thromboangiitis obliterans (initital news)," Przeglad lekarski, vol. 61, no. 12, pp. 1346-1350, 2004.

[44] J. H. Korn, M. Mayes, M. M. Cerinic et al., "Digital ulcers in systemic sclerosis: prevention by treatment with bosentan, an oral endothelin receptor antagonist," Arthritis and Rheumatism, vol. 50, no. 12, pp. 3985-3993, 2004.

[45] P. Garcia de la Peña-Lefebyre, S. R. Rubio, M. V. Expósito, L. Carmona, M. L. G. Gámir et al., "Long term experience of bosentan for treating ulcers and healed ulcers in systemic sclerosis patients," Rheumatology, vol. 47, no. 4, pp. 464-466, 2008.

[46] Y. Nagai, M. Hasegawa, T. Hattori, E. Okada, O. Tago, and O. Ishikawa, "Bosentan for digital ulcers in patients with systemic sclerosis," Journal of Dermatology, vol. 39, no. 1, pp. 48-51, 2012.

[47] J. A. R. Ivorra, C. P. Simeon, J. J. A. Sancho et al., "Bosentan in clinical practice for treating digital and other ischemic ulcers in Spanish patients with systemic sclerosis: IBER-DU cohort study," Journal of Rheumatology, vol. 38, no. 8, pp. 1631-1635, 2011.

[48] Y. Nagai, A. Shimizu, and O. Ishikawa, "Successful treatment with bosentan for refractory digital ulcers in a patient with systemic lupus erythematosus," Journal of Dermatology, vol. 35, no. 7, pp. 447-451, 2008.
[49] E. Suzuki, H. Kyozuka, T. Nishida, T. Kanno, and H. Ohira, "Systemic lupus erythematosus, complicated with refractory skin ulcers, treated successfully with bosentan," Modern Rheumatology, vol. 19, no. 4, pp. 447-451, 2009.

[50] S. Noda, Y. Asano, S. Masuda et al., "Bosentan: a novel therapy for leg ulcers in Werner syndrome," Journal of the American Academy of Dermatology, vol. 65, no. 2, pp. e54-e55, 2011.

[51] N. J. Carter and G. M. Keting, "Bosentan in pediatric patients with pulmonary arterial hipertensión," Pediatric Drugs, vol. 12, no. 1, pp. 63-73, 2010.

[52] M. A. González-Fernández and J. García-Consuegra, "Polyarteritis nodosa resistant to conventional treatment in a pediatric patient," Annals of Pharmacotherapy, vol. 41, no. 5, pp. 885-890, 2007.

[53] D. M. Wahezi, N. T. Ilowite, K. M. Kenney-Riley, and P. F. Belamarich, "Chronic leg ulceration as the presenting feature of diffuse systemic slcerosis in childhood," Journal of Pediatrics, vol. 159 , no. 4 , p. $698,2011$. 


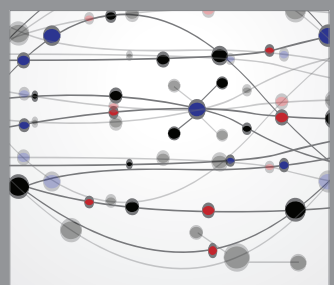

The Scientific World Journal
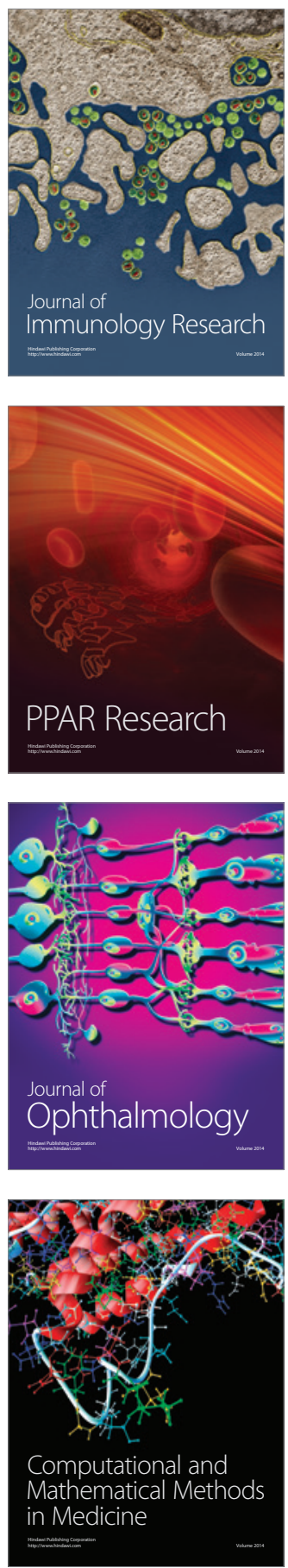

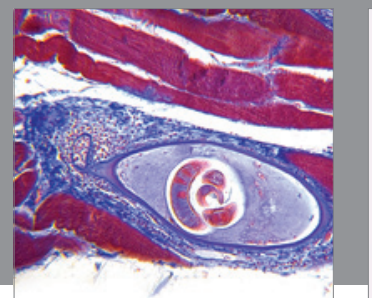

Gastroenterology

Research and Practice
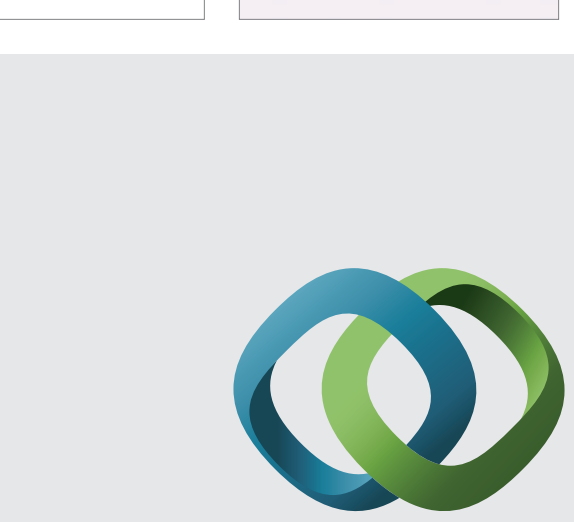

\section{Hindawi}

Submit your manuscripts at

http://www.hindawi.com
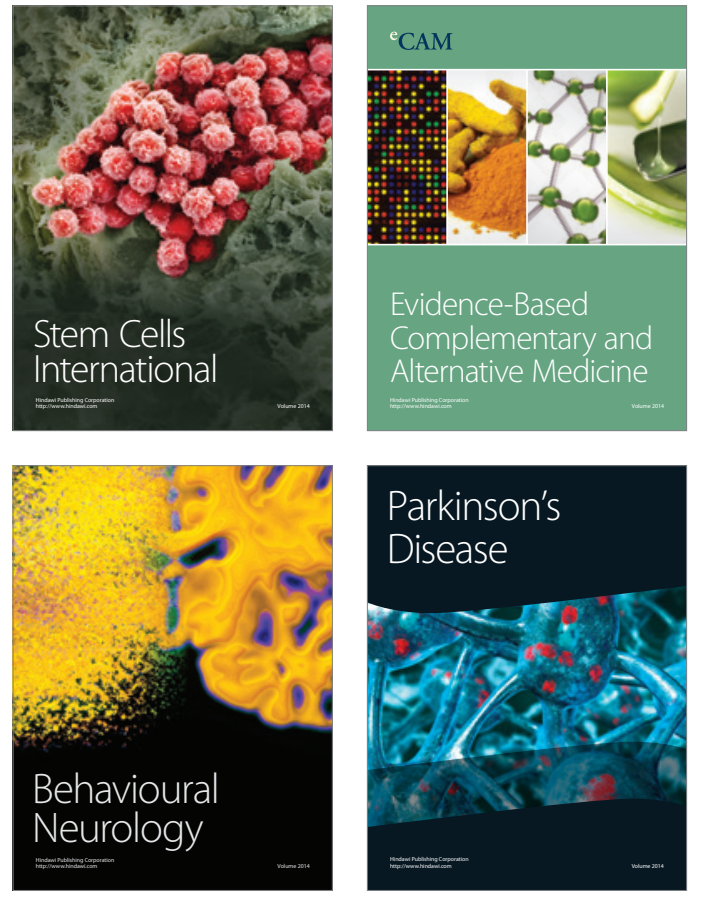
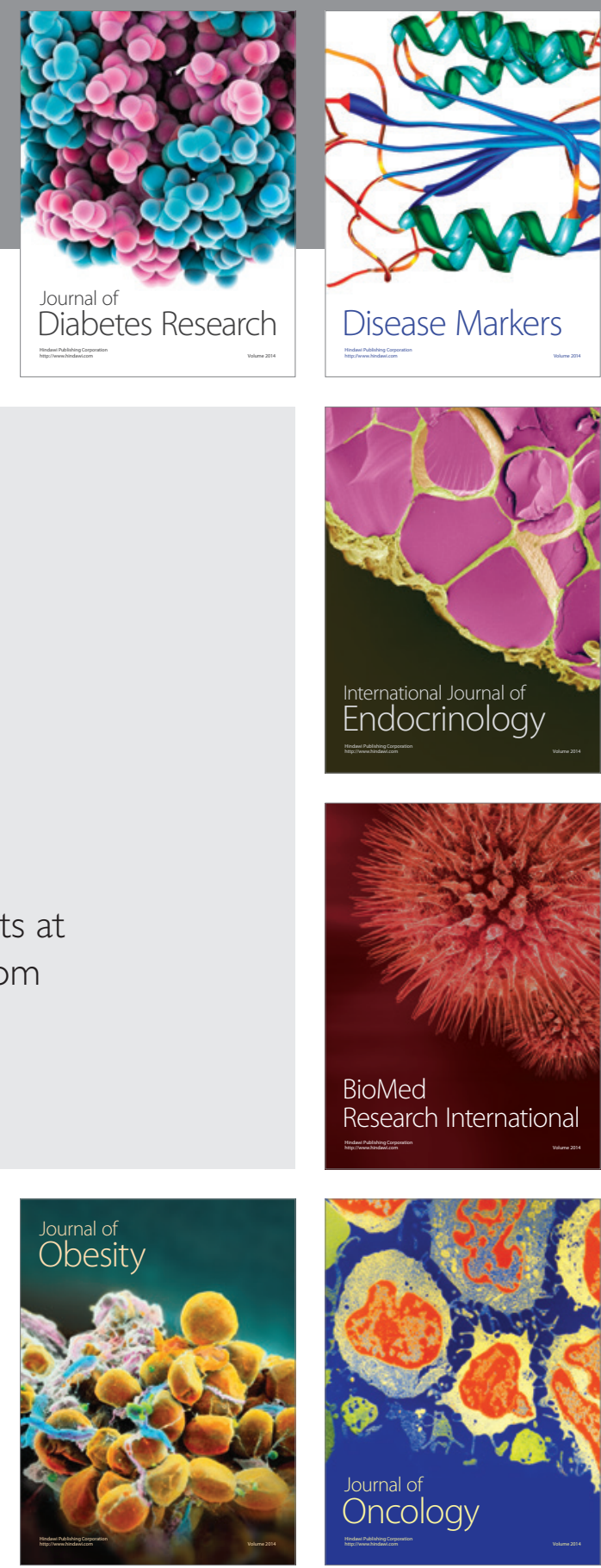

Disease Markers
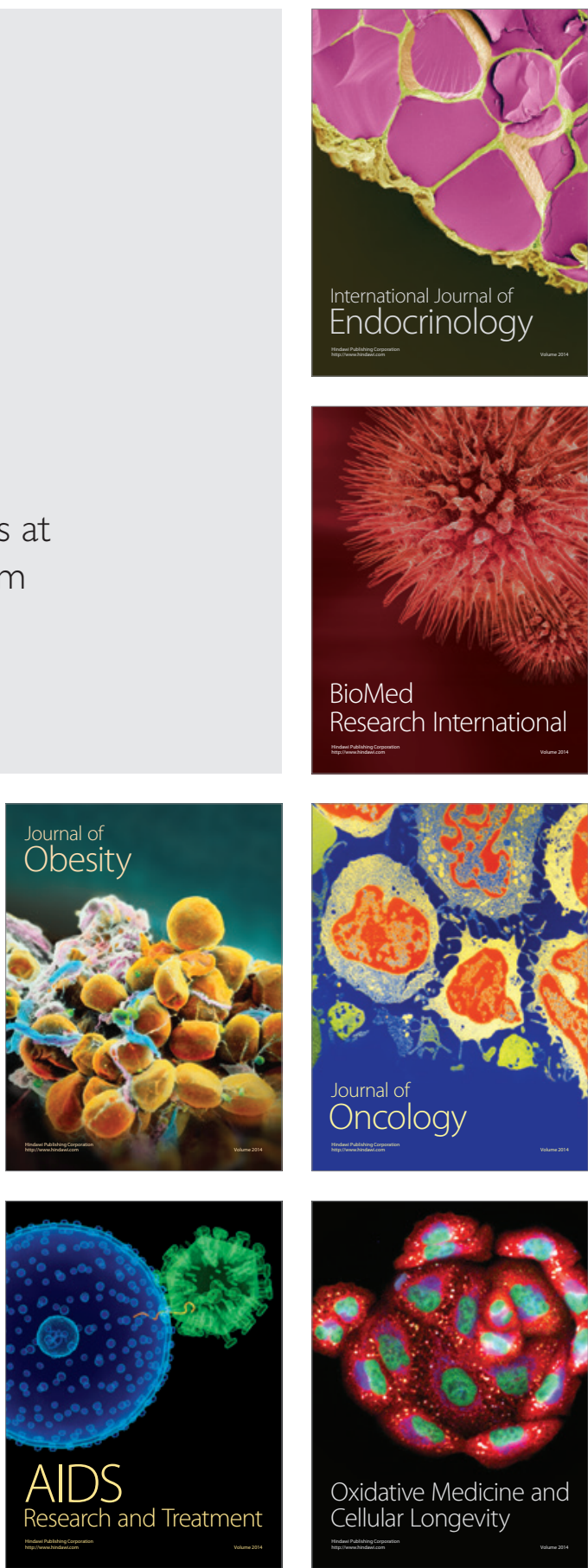